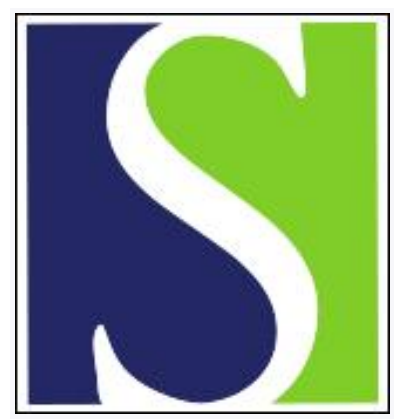

Scand J Work Environ Health 2002;28(2):85-93

https://doi.org/10.5271/sjweh.652

Issue date: Apr 2002

Effectiveness of a worksite exercise program with respect to perceived work ability and sick leaves among women with physical work

by Nurminen E, Malmivaara A, Ilmarinen J, Ylöstalo P, Mutanen P, Ahonen G, Aro T

Affiliation: Finnish Institute of Occupational Health, Department of Occupational Health Care, Topeliuksenkatu 41 a A, Fl-00250 Helsinki, Finland. eija.nurminen@ttl.fi

Refers to the following texts of the Journal: 1997;23 suppl 1:49-57 1997;23 suppl 1:66-71

The following articles refer to this text: $2002 ; 28(2): 73-74$; 2004;30(1):36-46; 2007;33(5):351-357; 2008;34(1):55-65; 2013;39(1):66-75; 2013;39(5):456-467; 2014;40(3):244-251; 2018;44(2):134-146

Key terms: absenteeism; female; intervention; job satisfaction; perceived work ability; physical activity; physical work; promotion; randomized controlled trial; self-assessment; self-report; sick leave; stress; woman; workplace; worksite exercise program

This article in PubMed: www.ncbi.nlm.nih.gov/pubmed/12019592

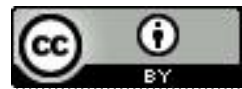




\title{
Effectiveness of a worksite exercise program with respect to perceived work ability and sick leaves among women with physical work
}

\author{
by Eija Nurminen, PhD, ${ }^{1}$ Antti Malmivaara, DrMedSci, ${ }^{1}$ Juhani IImarinen, PhD, ${ }^{1}$ Pekka Ylöstalo, MSc, ${ }^{1}$ \\ Pertti Mutanen, MSc, ${ }^{1}$ Guy Ahonen, PhD, ${ }^{1}$ Timo Aro, DrMedSci ${ }^{2}$
}

\begin{abstract}
Nurminen E, Malmivaara A, Ilmarinen J, Ylöstalo P, Mutanen P, Ahonen G, Aro T. Effectiveness of a worksite exercise program with respect to perceived work ability and sick leaves among women with physical work. Scand J Work Environ Health 2002;28(2):85-93.
\end{abstract}

\begin{abstract}
Objectives This multicentered randomized controlled trial evaluated the effect of worksite exercise intervention on perceived work ability and sick leaves.

Methods Women ( $\mathrm{N}=260$, mean age 40 years) engaged in physically demanding laundry work were individually randomized into an intervention $(\mathrm{N}=133)$ or control $(\mathrm{N}=127)$ group. Perceived work ability was assessed with questionnaires at $3,8,12$, and 15 months. Sick leave information was obtained from the personnel administration. Follow-up attendance was $100 \%$ at 3 months but declined gradually to $90 \%$ by 15 months. Both the intervention and control subjects received a 30-minute feedback on their physical capacity from a physiotherapist and individual exercise prescription and counseling. The intervention subjects also participated in worksite exercise training guided by a physiotherapist. Sixty-minute sessions $(\mathrm{N}=26)$ were held once a week for 8 months. About $50 \%$ of the intervention group participated in at least two-thirds of the sessions.

Results According to a dichotomized work ability index, at 12 months, workers with "good" or "excellent" work ability increased more in the intervention group than in the control group (11.0\%, 95\% CI 0.2-21.9), as did the health-related prognosis of work ability at 8 months $(8.1 \%, 95 \%$ CI $0.5-16.3)$. There were no statistically significant differences between the two groups as regards job satisfaction, work ability index (including series of questions on 7 items), or sick leaves.

Conclusions Physical activity once a week at worksites improves the perceived work ability of women with physically demanding work only slightly. Perceived work ability and sick leaves cannot be affected very positively using single-component exercise intervention. Work ability promotion may need a more multiprofessional approach.
\end{abstract}

Key terms absenteeism from work, female, intervention, job satisfaction, physical activity, promotion, randomized controlled trial, stress, workplace.

Worksite exercise programs have been carried out in various countries since the mid-1970s (1). The promotion of work ability has focused on the health and functional capacity of employees. Regular moderate exercise has been claimed to have both preventive and health-enhancing effects (2). Within the work ability concept, physical activity is one means with which to promote individual resources, although perceived work ability depends as well on many other individual characteristics and work-related factors (3). According to a randomized controlled trial carried out by Oden et al (4), aerobic exercise three times a week did not effectively increase job satisfaction among 45 blue-collar workers.

The fact that the Finnish workforce is aging must be taken into account with respect to workplace health promotion in Finland. Work ability is, to a considerable extent, based on physical capacity in physically demanding work, which, in itself, does not seem to prevent a decline in the work ability of aging men and women (5). Therefore, an adequate level of physical activity may be needed to maintain or promote work ability, in particular among aging workers (6). Preventive measures

1 Finnish Institute of Occupational Health, Helsinki, Finland.

2 Ilmarinen Mutual Pension Insurance Company, Helsinki, Finland.

Reprint requests to: Eija Nurminen, Finnish Institute of Occupational Health, Department of Occupational Health Care, Topeliuksenkatu 41 a A, FIN-00250 Helsinki, Finland. [E-mail: eija.nurminen@ttl.fi] 
that promote work ability should be started early enough, especially for workers in physically demanding jobs, to avoid, for example, early retirement (3).

From the point of view of occupational health care, musculoskeletal disorders that cause disability and absenteeism are a common and increasing problem among workers, and their occurrence may possibly be positively affected by exercise (7). In addition, although sick leave is a parameter that companies follow in business life, only a few randomized controlled trials involving workplace health promotion have used sick leave as an outcome. Three studies did, however, find that worksite exercise twice a week decreased sick leaves due to back pain (8-10). On the basis of this information, we undertook a randomized controlled trial using guided exercise intervention to evaluate the effectiveness of a worksite exercise program on perceived work ability and the occurrence of sick leave among women with physically demanding work. The study was integrated into a campaign promoting work ability among laundry workers through self-directed physical activity with the aim of increasing functional capacity and, eventually, improving work ability.

\section{Subjects and methods}

All the procedures used in this multicenter study complied with the Declaration of Helsinki and were approved by the Review Board and Ethics Committee of the Finnish Institute of Occupational Health.

\section{Participants, recruitment and data collection}

Women with physically demanding work who were permanently employed and without contraindications for physical capacity tests (respiratory inflammation, coronary heart disease, cardiac arrhythmia under physical strain, or other medical diseases) were included in the study. Sixty-five of 325 refused or did not fulfill the

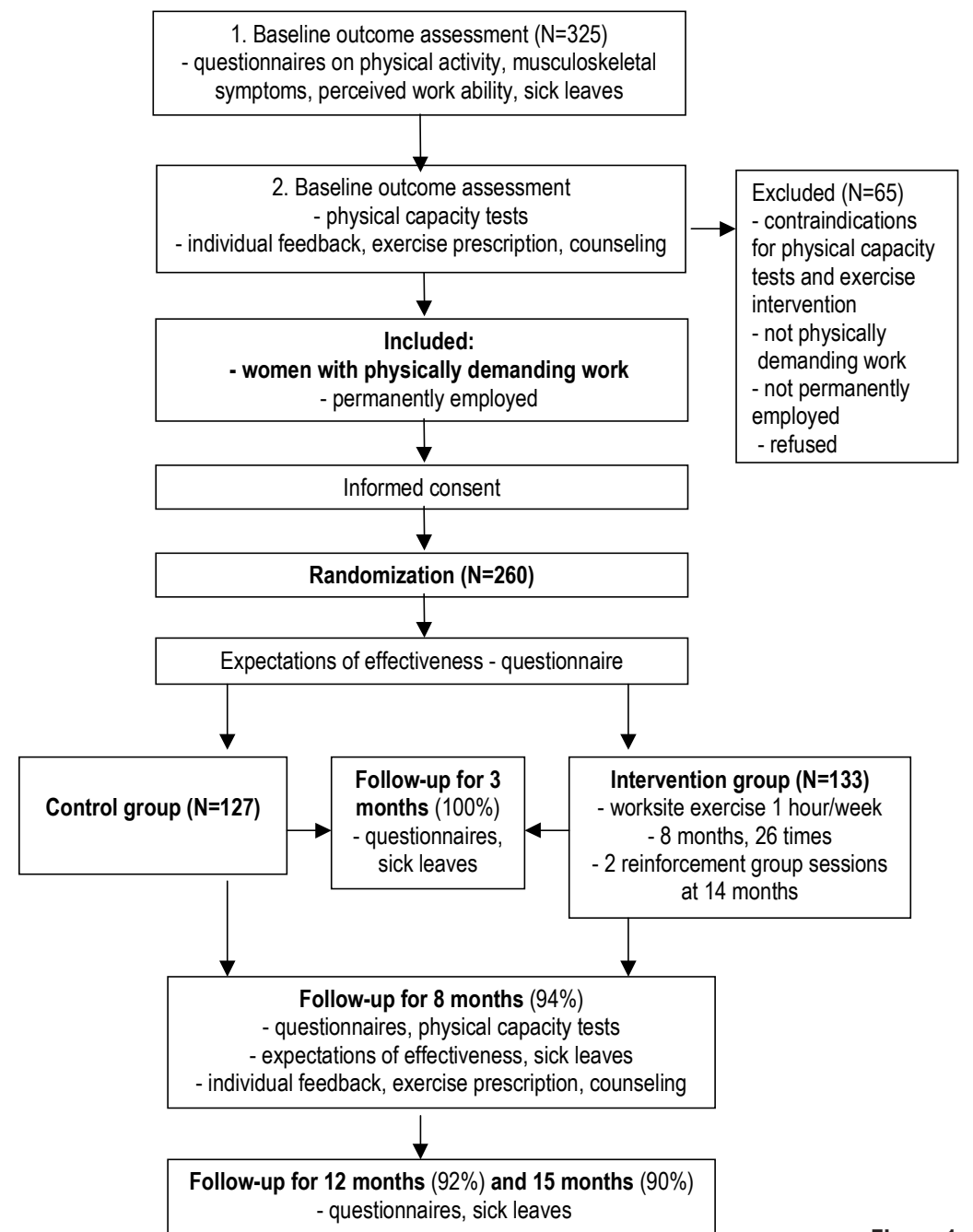

Figure 1. Flow of the study. 
inclusion criteria. Altogether 260 workers, aged 19 to 64 years, were therefore enrolled (figure 1).

The study protocol was implemented by specially trained occupational health care personnel, all of whom received written material and had phone contact with the co-ordinating principal researcher $(\mathrm{EN})$ when needed. In August 1996 the principal researcher visited all 11 production units of the laundry company in question to give a 1-hour presentation on work ability promotion and the forthcoming randomized control trial.

Data were collected in the following five phases: at the time of the baseline examination (in September 1996) and 3, 8, 12, and 15 months thereafter. Perceived work ability was assessed with structured questionnaires by occupational health nurses. The participants filled out the questionnaires in a group, during workhours, and returned them to the occupational health nurses, who sent the questionnaires to the co-ordinating researcher. All nonrespondents received one telephone reminder and up to two written reminders.

Prior to the randomization, and also 8 months thereafter, the participants and occupational health nurses and physiotherapists were asked to rate their expectations of the effectiveness of the contents of the intervention and control groups on a scale from 0 to 10 . At the 8-month follow-up, all the participants were also asked to grade their level of satisfaction regarding the contents of the intervention and control groups on a scale from 0 to 10.

\section{Outcome measures}

One of the primary outcomes, perceived work ability, was assessed using two questionnaires. First, work ability was assessed using the work ability index, which includes a series of questions dealing with seven items (6, 11, 12). The score ranges from 7 to 49 and is divided into the following four categories: poor (7-27 points), moderate (28-36 points), good (37-43 points), and excellent (44-49 points). Items of the index are (i) work ability compared with the lifetime best on a scale of 0 10 , (ii) work ability in relation to physical and mental demands of the work, (iii) number of diagnosed diseases, (iv) estimation of work impairment due to diseases, (v) self-reported sick leaves during the past 12 months, (vi) health-related prognosis of work ability on behalf of health after 2 years, and (vii) mental resources.

Second, perceived work ability was assessed using five questions that belong to a modified version of the Nordic questionnaire (13). The variables, ranging from 1 to 5 , were prognosis of work ability during the next 5 years with respect to musculoskeletal symptoms, health status compared with that of persons of the same age, stress, job satisfaction, and life satisfaction. Both questionnaires have been used nationwide and have gender-, work- and age-related reference values $(12,14)$. The variables that are based on single questions, the work ability index, as well as the index of mental resources are shown in table 1 .

Table 1. Demographic and perceived work ability characteristics of the participants at the time of the baseline examination.

\begin{tabular}{|c|c|c|}
\hline Characteristic & $\begin{array}{l}\text { Intervention group } \\
\quad(\mathrm{N}=133)\end{array}$ & $\begin{array}{l}\text { Control group } \\
(\mathrm{N}=127)\end{array}$ \\
\hline \multicolumn{3}{|l|}{ Demographic features } \\
\hline Age (year, mean) & 40.7 & 39.1 \\
\hline Married (\%) & 56.1 & 47.6 \\
\hline Intermediate or secondary education (\%) & 22.3 & 23.2 \\
\hline Vocational college or university education (\%) & 11.4 & 12.6 \\
\hline \multicolumn{3}{|l|}{ Perceived work ability } \\
\hline Work ability index (mean): 7-49 points/7 items & 40.0 & 39.8 \\
\hline Work ability (\%): good (37-43 points) or excellent (44-49 points) & 73.7 & 75.7 \\
\hline Work ability compared with lifetime best: scale 0-10 (mean) & 8.5 & 8.4 \\
\hline Work ability in relation to physical demands of the work (\%): very good or rather good & 76.7 & 77.4 \\
\hline Work ability in relation to mental demands of the work (\%): very good or rather good & 82.7 & 78.2 \\
\hline \multicolumn{3}{|l|}{ Health-related factors } \\
\hline Health status compared with that of persons of the same age (\%): very good or rather good & 69.0 & 64.0 \\
\hline Prognosis of work ability with respect to health after 2 years (\%): fairly sure able to do current job & 81.2 & 82.9 \\
\hline Prognosis of work ability during next 5 years with respect to musculoskeletal symptoms (\%): no difficulties & 59.1 & 60.3 \\
\hline Mental resources (mean): 3 items a & 9.5 & 9.3 \\
\hline \multicolumn{3}{|l|}{ Perceived well-being } \\
\hline Stress (\%): very much or rather much & 8.3 & 8.1 \\
\hline Job satisfaction (\%): very good or rather good & 75.6 & 71.8 \\
\hline Life satisfaction (\%): very good or rather good & 87.9 & 85.5 \\
\hline
\end{tabular}

a Enjoying daily tasks: 0 (never) - 4 (always), activity and life spirit: 0 (never) - 4 (always), and optimistic about future: 0 (never) -4 (continuously). 
The other primary outcome was information on sick leaves, concerning the years 1996 and 1997, obtained from the personnel administration. Sick leaves in the period January-August in 1996 formed the baseline data. Individual changes of sick leaves in the intervention and the control groups at 8 months (September 1996-May 1997), at 12 months (September 1996-August 1997), and at 15 months were compared with the time before randomization. The effectiveness of the intervention was assessed by calculating cumulative sick leaves due to any disease in the intervention and control groups. In addition, as subgroup analyses the between group differences in sick leaves were assessed among workers who had had sick leaves less than 10 days, over 10 days, and 10-30 days at the baseline.

\section{Randomization}

Concealment of treatment allocation was ensured by centralized randomization. Sequentially numbered, sealed opaque envelopes were sent to the occupational health care units. After the baseline assessment and informed consent, the 260 female laundry workers were individually randomized to the intervention $(\mathrm{N}=133)$ or control $(\mathrm{N}=127)$ group in 11 production units in various Finnish cities. An independent physiotherapist opened each envelope during the initial feedback session for each subject.

The study groups proved to be fairly comparable at the time of the baseline examination regarding demographic variables and outcome measures (table 1). The mean sick leave was 65.6 (SD 96.0) hours for the intervention group and 53.0 (SD 73.7) hours for the control group between January and August 1996.

\section{Intervention}

A physiotherapist gave every subject 30 minutes of feedback on the results of the physical capacity tests and also provided each subject with a prescription for exercise and counseling to increase their self-directed physical activity during leisure time. In addition, the intervention subjects participated as a group in worksite exercise guided by a physiotherapist. The sessions lasted 60 minutes once a week during workhours and totaled 26 sessions over an 8-month period (ie, 12 sessions in the autumn of 1996 and 14 in the spring of 1997). The adherence to the intervention and the mode of exercise were rated on a participation form that the physiotherapist filled out after each session. Two additional 60minute reinforcement sessions were arranged for the intervention group in the autumn of 1997 to promote physical activity.
The group sessions consisted of moderate worksite exercise based on a guidebook published by the Finnish Institute of Occupational Health for promoting work ability and physical activity through group exercise. The program involved muscle strengthening, cardiovascular exercise, and stretching. The exercise intensity increased progressively, the mean of the perceived exertion rating being 7.8 (SD 4.7) in the spring at the third exercise session and 8.6 (SD 4.4) at tenth session, on a scale of $0-10$ (15). The laundry company agreed not to arrange other campaigns promoting physical activity during the study.

\section{Recruitment, response rates, dropouts and adherence}

Eighty percent of the company's female employees engaged in physically demanding work participated in the study. Informed consent was obtained from all the participants. The proportion attending the follow-up was $100 \%$ at 3 months, $94 \%$ at 8 months, $92 \%$ at 12 months, and $90 \%$ at 15 months. Complete responses to the questionnaire concerning the work ability index (series of questions on 7 items) were received from 217 of the workers $(84 \%)$ by the time of the baseline measurements.

At the time of the 15-month follow-up, the dropouts from the intervention group $(\mathrm{N}=10)$ differed significantly from those of the control group ( $\mathrm{N}=18)$ with respect to baseline characteristics only regarding two outcomes: "very good" or "rather good" job satisfaction $(9.1 \%$ of the intervention group and $15.9 \%$ of the control group, $\mathrm{P}=0.02$ ) and "very good" or "rather good" perceived health status in comparison with that of other persons the same age $(10.8 \%$ of the intervention group and $16.6 \%$ of the control group, $\mathrm{P}=0.06$ ). In addition the dropouts from the intervention group (mean age 29 years) were also younger than those from the control group (mean age 36 years) at the time of the baseline assessments (table 1).

Seventy-five percent of the subjects in the intervention group participated in over half of the guided group exercise sessions, and about $50 \%$ took part in at least two-thirds of the sessions. The median of attendance was 9 (of 12 sessions) in the autumn and 10 (of 14 sessions) in the spring.

\section{Statistical analysis}

The power calculation showed a need for 35-97 subjects in both groups for the dichotomous variables, depending on the prevalence of phenomena in both groups, to detect a difference of $20 \%$ in the proportions in order to achieve a statistical power of 0.80 with an alpha of 0.05 (16). 
The data were analyzed according to the intentionto-treat principle. An analysis of covariance was performed to compare the groups (intervention versus control) for continuous variables (work ability index, mental resources index, work ability compared with the lifetime best on a scale of $0-10$, and sick leaves). A binomial regression model (using the identity link function to estimate the rate difference) was used for the dichotomous outcome variables. In addition, a nonparametric Wilcoxon rank sum test was used to compare the groups. The baseline value of each outcome variable was always included in the model in order to control for possible baseline differences.

The t-test was used to compare the expectations of the occupational health nurses and the physiotherapists with respect to the effectiveness of the two groups. In addition, the t-test and the Wilcoxon rank sum analysis test were used to compare the dropouts of the intervention group with the dropouts of the control group at the time of the 15-month follow-up according to their baseline data. The analyses were performed using SAS version 6.12 (17).

\section{Results}

Perceived work ability at the time of the 3-, 8-, 12- and 15-month follow-up

The health-related prognosis of work ability after 2 years had improved significantly after 8 months. The proportions of workers with "good" or "excellent" work ability had increased more in the intervention group than in the control group at the time of the 12-month followup, and the trend was positive also at 3 and 8 months. However, work ability in relation to the mental demands of the work had improved statistically significantly more in the control group after 3 months. There were no statistically significant differences in the other outcomes between the two groups (tables 2 and 3).

\section{Sick leaves at 8, 12 and 15 months}

Cumulative sick leave (in hours) at the time of the 8-, 12 -, and 15-month follow-ups was 87,108 , and 154 hours, respectively, for the intervention group and 82 ,

Table 2. Perceived work ability at the time of the 3- and 8-month follow-ups, presented as the baseline-adjusted differences in the means and proportions (\%) between the intervention and control groups (effectiveness), $95 \%$ confidence intervals (95\% Cl), and the P-values for the group differences. A positive effectiveness value stands for an advantage of the intervention group and a negative value for an advantage of the control group.

\begin{tabular}{|c|c|c|c|c|c|c|c|c|}
\hline \multirow{3}{*}{ Perceived work ability } & \multicolumn{4}{|c|}{ Three-month follow-up } & \multicolumn{4}{|c|}{ Eight-month follow-up } \\
\hline & \multirow[t]{2}{*}{ Effectiveness } & \multirow[t]{2}{*}{ s $95 \% \mathrm{Cl}$} & P-value ${ }^{a}$ & P-value $2^{b}$ & Effectiveness & s $95 \% \mathrm{Cl}$ & \multicolumn{2}{|c|}{ P-value $1^{a} P$-value $2^{b}$} \\
\hline & & & & & & & & \\
\hline Work ability index (mean): 7-49 points/7 items & 0.5 & $-0.6-1.7$ & 0.37 & 0.66 & 0.5 & $-0.7-1.7$ & 0.37 & 0.50 \\
\hline $\begin{array}{l}\text { Work ability (\%): good (37-43 points) or excellent } \\
\text { (44-49 points) }\end{array}$ & 9.3 & $-0.5-18.5$ & 0.07 & & 9.9 & $-0.6-20.6$ & 0.06 & \\
\hline $\begin{array}{l}\text { Work ability compared with the lifetime best: } \\
\text { scale } 0 \text { - } 10 \text { (mean) }\end{array}$ & 0.1 & $-0.2-0.4$ & 0.38 & 0.87 & 0.1 & $-0.3-0.5$ & 0.56 & 0.64 \\
\hline $\begin{array}{l}\text { Work ability in relation to physical demands of the } \\
\text { work (\%): very good or rather good }\end{array}$ & 1.4 & $-8.1-10.9$ & 0.78 & 0.44 & -1.7 & $-11.0-7.6$ & 0.72 & 0.77 \\
\hline $\begin{array}{l}\text { Work ability in relation to mental demands of the } \\
\text { work (\%): very good or rather good }\end{array}$ & -4.4 & $-14.1-5.2$ & 0.37 & 0.02 & -4.1 & $-13.4-5.1$ & 0.38 & 0.08 \\
\hline Health-related factors & & & & & & & & \\
\hline $\begin{array}{l}\text { Health status compared with that of persons of the } \\
\text { same age }(\%) \text { : very good or rather good }\end{array}$ & -5.7 & $-14.8-3.3$ & 0.22 & 0.27 & 5.4 & $-2.6-13.6$ & 0.18 & 0.21 \\
\hline $\begin{array}{l}\text { Prognosis of work ability with respect to health after } \\
2 \text { years (\%): fairly sure able to do current job }\end{array}$ & 7.0 & $-0.2-14.9$ & 0.06 & 0.10 & 8.1 & $0.5-16.3$ & 0.041 & 0.054 \\
\hline $\begin{array}{l}\text { Prognosis of work ability during the next } 5 \text { years with } \\
\text { respect to musculoskeletal symptoms (\%): no difficulties }\end{array}$ & -4.4 & $-14.3-5.6$ & 0.38 & 0.88 & 1.6 & $-9.2-12.3$ & 0.78 & 0.55 \\
\hline Mental resources (mean): 3 items ${ }^{c}$ & -0.3 & $-0.7-0.1$ & 0.14 & 0.22 & 0.2 & $-0.2-0.6$ & 0.43 & 0.72 \\
\hline Perceived well-being & & & & & & & & \\
\hline Stress (\%): very much or rather much & 3.2 & $-3.4-10.0$ & 0.33 & 0.76 & -4.5 & $-10.4-0.7$ & 0.09 & 0.90 \\
\hline Job satisfaction (\%): very good or rather good & -2.2 & $-11.2-6.6$ & 0.62 & 0.46 & -5.2 & $-13.3-2.8$ & 0.18 & 0.21 \\
\hline Lif satisfaction (\%): very good or rather good & -0.3 & $-6.3-5.8$ & 0.91 & 0.62 & -0.6 & $-5.2-4.2$ & 0.79 & 0.41 \\
\hline
\end{tabular}

a Binomial regression model for dichotomous variables or an analysis of covariance for continuous variables.

${ }^{b}$ Wilcoxon rank sum test for the continuous and original ordinal variables.

c Enjoying daily tasks: 0 (never) - 4 (always), activity and life spirit: 0 (never) - 4 (always), and optimistic about future: 0 (never) - 4 (continuously). 
Table 3. Perceived work ability at the time of the 12- and 15-month follow-ups, presented as the baseline-adjusted differences in the means and proportions (\%) between the intervention and control groups (effectiveness), $95 \%$ confidence intervals (95\% Cl), and the Pvalues for the group differences. A positive effectiveness value stands for an advantage of the intervention group and a negative value represents an advantage for the control group.

\begin{tabular}{|c|c|c|c|c|c|}
\hline 12-month foll & ow-up & & 15-month follo & w-up & \\
\hline Effectiveness & $95 \% \mathrm{Cl}$ & $\mathrm{P}$-value ${ }^{\mathrm{a}} \mathrm{P}$-value ${ }^{\mathrm{b}}$ & Effectiveness & $95 \% \mathrm{C}$ & P-value $1^{\text {a }} \mathrm{P}$-value $2^{\mathrm{b}}$ \\
\hline
\end{tabular}

\section{Perceived work ability}

Work ability index (mean): 7-49 points/7 items

Work ability (\%): good (37-43 points) or excellent (44--49 points)

Work ability compared with the lifetime best: scale $0-10$ (mean)

Work ability in relation to the physical demands of the work (\%): very good or rather good

Work ability in relation to the mental demands of the work (\%): very good or rather good

\section{Health-related factors}

Health status compared with that of persons of the same age $(\%)$ : very good or rather good

Prognosis of work ability with respect to health after 2 years (\%): fairly sure able to do current job

Prognosis of work ability during the next 5 years with respect to musculoskeletal symptoms (\%): no difficulties

Mental resources (mean): 3 items ${ }^{c}$

Perceived well-being

Stress (\%): very much or rather much Job satisfaction (\%): very good or rather good Life satisfaction (\%): very good or rather good

$\begin{array}{rrrrrrrr}0.02 & -1.51-1.47 & 0.98 & 0.87 & -0.01 & -1.4-1.4 & 1.00 & 1.00 \\ 11.0 & 0.2-21.9 & 0.04 & & 1.1 & -10.7-13.1 & 0.85 & \\ 0.11 & -0.24-0.47 & 0.53 & 0.42 & 0.04 & -0.3-0.4 & 0.83 & 0.84 \\ 5.3 & -4.1-15.0 & 0.27 & 0.44 & 6.6 & -2.8-16.4 & 0.17 & 0.31 \\ -3.0 & -13.1-7.1 & 0.56 & 0.07 & -0.5 & -11.2-10.1 & 0.92 & 0.26\end{array}$

$-4.5-11.2 \quad 0.40$

0.74

1.8

$-7.9-11.7 \quad 0.71$

0.39

7.4

$-1.1-16.30 .09$

0.14

2.5

$-5.2-10.7 \quad 0.53$

0.64

4.2

$\begin{array}{ll}-7.1-15.5 & 0.47\end{array}$

0.06

4.0

$\begin{array}{ll}-8.0-15.9 & 0.52\end{array}$

0.18

$-0.1$

$-0.6-0.3 \quad 0.62$

0.77

$-0.18$

$-0.7-0.3 \quad 0.46$

0.32

$\begin{array}{rrrrrrll}-1.7 & -9.0-5.2 & 0.62 & 0.63 & -5.5 & -13.6-1.9 & 0.15 & 0.43 \\ -0.7 & -10.4-8.9 & 0.88 & 0.99 & 3.7 & -7.4-15.0 & 0.51 & 0.6 \\ 4.0 & -9.9-1.6 & 0.14 & 0.84 & 1.2 & -6.2-8.9 & 0.75 & 0.78\end{array}$

a Binomial regression model for dichotomous variables or an analysis of covariance for continuous variables.

${ }^{b}$ Wilcoxon rank sum test for the continuous and original ordinal variables.

c Enjoying daily tasks: 0 (never) - 4 (always), activity and life spirit: 0 (never) - 4 (always), and optimistic about future: 0 (never) - 4 (continuously).

Table 4. Baseline-adjusted differences (hours) in cumulative sick leave between the intervention and the control groups at the time of the 8-, 12-, and 15-month follow-ups, and the 95\% confidence intervals $(95 \% \mathrm{Cl})$. A positive effectiveness value stands for an advantage of the control group.

\begin{tabular}{rcc}
\hline & Effectiveness (h) & $95 \% \mathrm{Cl}$ \\
\hline 8-month follow-up & 5.7 & $-16.9-28.3$ \\
12-month follow-up & 12.0 & $-15.8-39.7$ \\
15-month follow-up & 22.5 & $-13.8-58.8$ \\
\hline
\end{tabular}

96, and 132 hours, respectively, for the control group. There were no statistically significant differences in the cumulative amount of sick leave between the intervention and control groups at any follow-up time (table 4).

There were no statistically significant differences in cumulative sick leave at the time of the 8-, 12-, or 15month follow-up when the effectiveness was assessed by the workers who had had sick leave less than 10 days, over 10 days, and 10-30 days at the time of the baseline measurements.

\section{Expectations of effectiveness}

According to a $0-10$ scale, both before and after the exercise program, the occupational health nurses and physiotherapists involved in the study expected that the intervention would be more effective than the program (measurements and initial feedback) that the control group received. The mean expectations of the intervention versus the control program were 7.7 (SD 0.7) and 5.7 (SD 1.6), respectively $(\mathrm{P}=0.0001)$ at the time of the baseline examination. At the end of the 8-month exercise intervention the means of the expectations were 7.3 (SD 1.3) and 5.7 (SD 1.7), respectively ( $\mathrm{P}=0.0005)$. The subjects in the intervention group also expected the effectiveness of their program to be higher both at the time of the baseline measurements (mean 7.2, SD 2.1) and after the intervention (mean 7.1, SD 2.3) than the control group (mean 6.4, SD 2.5, and 5.5, SD 2.6, respectively) did.

The subjects in the intervention group were also more satisfied with their exercise program (mean 8.0, 
SD 2.1, $\mathrm{N}=126$ ) at the end of the intervention than the controls (mean 6.1, SD 2.4, $\mathrm{N}=108$ ) were.

\section{Discussion}

\section{Study design}

Although primary preventive worksite exercise programs are widely used, evidence of their effectiveness with respect to perceived work ability and sick leaves is scarce. So far randomized controlled trials have proved to offer the most valid study design with which to produce generalized information on the effectiveness of an intervention. However, few such trials have been carried out in the field of workplace health promotion, probably because of the practical difficulties involved. The aim of this randomized controlled trial was to assess the effectiveness of a primary preventive worksite exercise program with regard to perceived work ability and sick leaves. Occupational health care personnel executed the study protocol at the workplaces, and the implementation proved to be feasible.

The subjects comprised $80 \%$ of all women engaged in physical laundry work in the company in question, and thus the sample can be considered fairly representative. The mean age of the participants was 40 years, and the laundry work was physically demanding for all of them, the load on the musculoskeletal system being high due to the repetitive lifting tasks and static postures. Therefore, intervention through physical activity seemed warranted since heavy physical work in itself does not seem to prevent a decline in the work ability of aging men and women (5) and improving the physical functional capacity of the workers may have improved their work ability.

Physiologically, 1 hour of exercise during workhours on a weekly basis is inadequate to improve physical fitness, but, for the company, it is a moderately costly investment due to lost production time and additional health care personnel costs. In addition, such interventions have been carried out without scientific evidence of any effectiveness.

The contents of the physical exercise intervention were aimed at promoting self-directed physical activity and increasing physical functional capacity. Results of outcomes related to physical activity and functional capacity are currently under submission. An important question related to health-enhancing exercise is how to promote self-directed, long-term adherence to physical activity in order to keep the dose sufficient. In our study the rate of attendance in physical exercise intervention at the workplace was moderately good, probably because it was arranged during workhours. Full adherence over a long term is hardly possible, however. Nevertheless, it seems that, for physical activity to increase, more resources or other methods (eg, support) are needed.

Our statistical analyses were performed with parametric and nonparametric methods (the original ordinal variables ranging from 1 to 5). Original ordinal variables are more sensitive to changes than dichotomous variables are. On the other hand, binomial regression is a more flexible modeling approach (eg, baseline values can be included in the model). If significant changes occur in both analyses, the results are more reliable than if only an ordinal or dichotomous variable shows significant change between the groups. With the use of accurate statistical analyses, the effectiveness in this randomized controlled trial was less than many before-after comparisons have shown.

\section{Interpretation of the findings on perceived work ability}

According to our study, physical exercise once a week at the workplace improves perceived work ability slightly among women with physically demanding work. At the end of 8 months of intervention, the health-related subjective prognosis for work ability was in the advantage of the intervention group. This result is relevant because subjective prognosis is regarded as an important predictor for, for example, return to work from sick leave. Besides, according to the dichotomized work ability index, the proportions of workers with "good" or "excellent work ability" had increased more in the intervention group than in the control group at the time of the 12-month follow-up. The favorable effects occurred mostly during the 8-month exercise intervention or shortly afterwards, the one exception occurring at the 3-month follow-up, when the subjects in the intervention group had lower "work ability in relation to the mental demands of the work" than those in the control group. This finding may be explained by chance, or possibly a new intervention caused mental load before the actions became integrated into normal daily routines. It therefore seems worthwhile to make the effort needed to implement actions promoting work ability properly.

The intervention effects on perceived work ability were modest when compared with the high expectations and good satisfaction of the participants in the intervention group and the occupational health care personnel before and after the intervention. Our study supports the findings of Oden et al (4) that weekly exercise is not effective in increasing, for example, job satisfaction.

At the baseline, the mean score of the work ability index was good (40 out of 49 points) among the participants, and this high score may explain the result of no difference between the groups. In previous studies among Finnish women over 50 years of age and engaged in physically demanding work, the index score, derived 
from a series of questions on seven items, has been about 34 (12). If we had enrolled only women with a low perceived work ability, it might have been easier to detect a more positive intervention effect. Our exercise intervention represented preventive workplace health promotion, and therefore the work ability index did not change much during the 15-month follow-up.

\section{Interpretation of the findings regarding sick leaves}

The 8-month intervention did not have a favorable effect on sick leaves. The effectiveness with respect to cumulative sick leave at 8-, 12-, and 15-months was, interestingly, in favor of the control group, although the differences between the two groups were not statistically significant. This result is contradictory to the findings of three earlier studies $(8,9,10)$, which showed some evidence that exercise twice a week for 18 months, 13 months, and 3 months decreased sick leaves due to back pain.

In this study the information on sick leaves was obtained from the personnel administration. We did not rely on self-reported data, which can be considered a strength of our study, because information from questionnaires is less precise and more liable to bias. Unfortunately the data from the personnel office did not include the sick-leave diagnoses, and therefore our interpretation of the sickness absence data is hampered. Sick leaves themselves do not indicate the reason for absenteeism.

\section{Limitations of the study}

Awareness of the intervention might have led to an increase in exercise among the subjects in the control group and thus diluted the contrast between the intervention and control groups. For ethical reasons the control group had to be able to participate in the company's campaign to promote health and work ability, although it was agreed with the company that no extra effort in this respect would be initiated among the employees during the follow-up. Nevertheless, previous studies have found the kind of intervention received by the control group (baseline assessments, individual feedback, exercise prescription, and counseling) comparable with the use of placebos with respect to increasing physical activity $(18,19)$.

Subjects in the intervention group and the occupational health care personnel had high expectations for the exercise program both before and after the intervention. These expectations may have biased the results based on subjective judgment (questionnaire data) towards an overestimation of the effects; nevertheless, no long-term effectiveness was found with respect to the work ability index and sick leaves. On the other hand, the aforementioned measures do not seem to be particularly sensitive to small changes during a preventive intervention program on the short term, especially when the mean age of the study population is as low as 40 years and the perceived work ability is rather good to begin with.

\section{Implications for practice}

The present study illustrated the feasibility of an individually randomized controlled trial at the workplace. The results can be considered when more effective means for promoting work ability within occupational health care are planned. Guided worksite exercise once a week improves perceived work ability only slightly among women with physically demanding work. It can be concluded that, on the long-term, single-component 8-month exercise intervention does not seem to yield very favorable results with respect to improving perceived work ability or decreasing sick leaves, which are complicated phenomena and partly depend on economic conjunctures as well.

However, regular physical exercise at a moderate level has preventive and health-enhancing effects (2). Therefore physical activity is an important part of workplace health promotion. Whether more intense and more frequent exercise will improve the health and work ability of aging women in physically demanding work is an open question. On the other hand, broader actions to promote work ability, including organizational and professional competence and environmental intervention with a multiprofessional approach, may be needed to improve job satisfaction and mental well-being at work in general. Although, from the scientific point of view, multidisciplinary interventions undertaken simultaneously are vulnerable to bias.

The aging of the workforce will affect many industrialized European countries in forthcoming years. In order to prevent, for example, early retirement, preventive promotion of health and work ability is needed. Optimally the scarce resources available would be directed towards evidence-based interventions. Before workplace health and work ability promotion is integrated into everyday practice, more efforts and valid research is clearly required on this topic.

\section{Acknowledgments}

This study was financially supported by the Lindström Company, the Finnish Institute of Occupational Health, the Finnish Work Environment Fund, the Swedish School of Economics and Business Administration, and the Ilmarinen Mutual Pension Insurance Company. 
We thank all the staff of the Lindström Company and the personnel of the occupational health care centers involved in the study for their help with the physical capacity testing, the questionnaires, and the worksite exercise program. Their commitment made it possible to implement the study protocol at the workplace. We also express our special thanks to the management of the Lindström Company for their positive attitude towards scientific research.

This randomized controlled trial has been reported in Finnish in four peer-reviewed articles, all published in the research series People and Work (report no 18), by the Finnish Institute of Occupational Health, Helsinki, in 2000. The report also includes, in Finnish, a systematic literature review of the effectiveness of worksite exercise as part of the doctoral dissertation of Eija Nurminen.

\section{References}

1. Shephard RJ. Worksite fitness and exercise programs: a review of methodology and health impact. Am J Health Promot 1996;10(6):436-52.

2. US Department of Health and Human Services. Physical activity and health: a report of the Surgeon General. Atlanta (GA): US Department of Health and Human Services, Centers for Disease Control and Prevention, National Center for Chronic Disease Prevention and Health Promotion 1996.

3. Ilmarinen J. Ageing workers in the European Union - status and the promotion of work ability, employability and employment. Helsinki: Finnish Institute of Occupational Health, Ministry of Social Affairs and Health, \& Ministry of Labour, 1999.

4. Oden G, Crouse S, Reynolds C. Worker productivity, job satisfaction, and work related stress; the influence of an employee fitness program. Fitness Business 1989:198-204.

5. Nygård C-H, Luopajärvi T, Ilmarinen J. Musculoskeletal capacity and its changes among aging municipal employees in different work categories. Scand J Work Environ Health 1991;17 suppl 1:110-7.

6. Smolander J, Blair SN, Kohl HW, 3rd. Work ability, physical activity, and cardiorespiratory fitness: 2-year results from Project Active. J Occup Environ Med 2000;42(9):906-10.

7. van Tulder M, Malmivaara A, Esmail R, Koes B. Exercise therapy for low back pain: a systematic review within the framework of the Cochrane Collaboration back review group. Spine 2000;25(21):2784-96.

8. Kellett KM, Kellett DA, Nordholm LA. Effects of an exercise program on sick leave due to back pain. Phys Ther 1991; 71(4):283-93.

9. Gundewall B, Liljeqvist M, Hansson T. Primary prevention of back symptoms and absence from work: a prospective randomized study among hospital employees. Spine 1993; 18(5):587-94.

10. Soukup MG, Glomsrod B, Lonn JH, Bo K, Larsen S. The effect of a Mensendieck exercise program as secondary prophylaxis for recurrent low back pain: a randomized, controlled trial with 12-month follow-up. Spine 1999;24 (15):1585-92.

11. Ilmarinen $\mathrm{J}$, Tuomi $\mathrm{K}$, Klockars $\mathrm{M}$. Changes in the work ability of active employees over an 11-year period. Scand J Work Environ Health 1997;23 suppl 1:49-57.

12. Tuomi K, Ilmarinen J, Jahkola A, Katajarinne L, Tulkki A. Work ability index. 2nd ed. Helsinki: Finnish Institute of Occupational Health, 1998.

13. Kuorinka I, Jonsson B, Kilbom A, Vinterberg H, BieringSorensen F, Anderson G. Standardized Nordic questionnaires for the analysis of musculoskeletal symptoms. Appl Ergon 1987(18):233-7.

14. Tuomi K, Ilmarinen J, Seitsamo J, Huuhtanen P, Martikainen R, Nygard CH, et al. Summary of the Finnish research project (1981-1992) to promote the health and work ability of aging workers. Scand J Work Environ Health 1997;23 suppl 1:6671.

15. Borg G, Ljunggren G, Ceci R. The increase of perceived exertion, aches and pain in the legs, heart rate and blood lactate during exercise on a bicycle ergometer. Eur J Appl Physiol 1985;54(4):343-9.

16. Campbell MJ, Julious SA, Altman DG. Estimating sample sizes for binary, ordered categorical, and continuous outcomes in two group. BMJ 1995;311(7013):1145-8. comparisons [published erratum appears in BMJ 1996;312(7023):96]

17. SAS Institute Inc. SAS/STAT Software. In: Changes and enhancements through Release 6.11. Cary (NC): SAS Institute Inc., 1996:1104.

18. Cardinal BJ, Sachs ML. Prospective analysis of stage-ofexercise movement following mail- delivered, self-instructional exercise packets. Am J Health Promot 1995;9(6):430 2.

19. Ostwald SK. Changing employees' dietary and exercise practices: an experimental study in a small company. J Occup Med 1989;31(2):90-7.

Received for publication: 25 June 2001 